Norman Kasper, Jana Kittelmann, Jochen Strobel und Robert Vellusig

\title{
Geschichte und Geschichtlichkeit des Briefs. Zur Einführung
}

\section{Die einzige Geschichte des deutschen Briefs}

In einer Fußnote seiner viel beachteten Fallstudien zum Briefschreiber Goethe hat Albrecht Schöne angemerkt, dass Georg Steinhausens Geschichte des deutschen Briefes die „einzige, immer noch förderliche“1 Geschichte des deutschen Briefes sei. Sie ist, wie Schönes Beistrich zu verstehen gibt, nicht nur die einzige Geschichte des Briefes, die immer noch förderlich ist, sondern die einzige Geschichte des Briefes überhaupt. Angesichts der Intensität, mit der in den letzten Jahrzehnten über den Brief nachgedacht wurde und mit der Briefkorpora editorisch erschlossen werden, angesichts der Tatsache auch, dass die Briefforschung inzwischen ausgesprochen beachtlichen Handbuchstatus ${ }^{2}$ erreicht hat und damit ins Stadium ihrer Selbstbilanzierung eingetreten ist, ist Schönes Befund eine Provokation.

Steinhausens Geschichte des deutschen Briefes stammt nicht nur aus dem neunzehnten Jahrhundert, sondern schließt auch dort: „Meine Darstellung von der Geschichte des deutschen Briefes ist zu Ende: in unseren Tagen durfte am ehesten eine solche versucht werden: denn seine eigentliche Geschichte liegt hinter uns, und es scheint, als ob es mit einer weiteren Entwickelung überhaupt vorbei sei.“3 Tatsächlich ist auch der Briefforschung des zwanzigsten Jahrhunderts der Gedanke nicht fremd, dass sich die Geschichte des Briefes im neunzehnten Jahrhundert vollendet hat. „Die Möglichkeiten des Mediums Brief“, so heißt es bei Johannes Anderegg, „sind kurz nach 1800 weitgehend ausgelotet“. ${ }^{4}$ Diese Behauptung ist zwar nicht unwidersprochen geblieben - Jörg Schuster hat in seiner ambitionierten „Kulturpoetik des Briefs“ den ,innovativen Charakter des Briefs in der Moderne“5

\footnotetext{
1 Schöne 2015, 16.

2 Vgl. Matthews-Schlinzig et al. 2020.

3 Steinhausen 1889, 410.

4 Anderegg 2000, 12.

5 Schuster 2014, 24.
}

Norman Kasper, Jana Kittelmann, Jochen Strobel und Robert Vellusig, Martin-Luther-Universität Halle-Wittenberg, Philipps-Universität Marburg, Karl-Franzens-Universität Graz

Ә Open Access. (c) 2021 Norman Kasper, Jana Kittelmann, Jochen Strobel, Robert Vellusig, publiziert von De Gruyter (cc) BY-NC-ND Dieses Werk ist lizenziert unter der Creative Commons Attribution-Non-CommercialNoDerivatives 4.0 International Lizenz. https://doi.org/10.1515/9783110712568-001 
zur Geltung gebracht -, die Frage aber, ob es eine Geschichte des Briefs überhaupt gibt und wie sie sich modellieren ließe, steht weiterhin zur Debatte.

Georg Steinhausens Briefgeschichte ist nationale Kulturgeschichtsschreibung ,bottom up': Nicht in den Haupt- und Staatsaktionen der politischen Geschichte, die der Historismus gepflegt hatte, sondern in der Kultur- und Wirtschaftsgeschichte, wie sie der Historiker Karl Lamprecht vertrat, sollte die Entstehung der Nation nachgezeichnet werden. Ihren materiellen Zeugnissen wurde dabei besondere Aufmerksamkeit zuteil - hierzu zählte Steinhausen auch Briefe. Ihm ging es darum, Briefe als „Vermittler der Geselligkeit“6 auf dem Weg der Deutschen zur Nationsbildung aufzuspüren. Briefe galten ihm als die Quellen, die den Zugang zum Innersten des ganzen Volkes eröffneten. Der Stil eines Volkes - nach Heinrich Laube sein „Kulturgesicht" "7 - wird für Steinhausen im Brief greifbar. Das achtzehnte Jahrhundert ernennt er zum „Jahrhundert des Briefes“. ${ }^{8}$ Mangels anderer (z.B. epochaler) Einteilungskriterien bietet die Gliederung in Jahrhunderte die Chance, Entwicklung als Steigerung in ganz groben Zügen vorzuführen.

Der Germanist Reinhard Nickisch unternahm 1991 einen weiteren Versuch

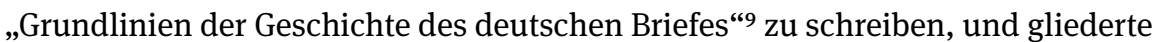
seine knappe Darstellung ebenfalls in Jahrhundertabschnitte. Nickisch erklärte das achtzehnte und das neunzehnte zu Jahrhunderten des Briefs. ${ }^{10}$ Die Entfaltung des ,persönlichen' Briefs, des säkular-empfindsamen Privatbriefs, gilt ihm als Wendepunkt der Briefgeschichte, als deren Ursachen er ,wirtschafts- und gesellschaftsgeschichtliche[] Prozesse[] des 18. Jhs.“" ${ }^{11}$ sowie ein „neu gewonnene[s] Selbstwertgefühl“ des deutschen Bürgertums identifiziert: „Das bürgerliche Ich begann, sich, seine Empfindungen und Gedanken wichtig zu nehmen, es beobachtete sich selbst, und es mußte sich mitteilen“.12

Die vorliegenden eineinhalb Geschichten des deutschen Briefes sind - wie sollte es anders sein - Kinder ihrer Zeit. Eine Monumentalgeschichte des Briefes, die die Genese des Volksgeistes nachzeichnet, steht nicht mehr zur Debatte; die Geschichte vom aufsteigenden Bürgertum hat sich inzwischen als Mythos des neunzehnten Jahrhunderts erwiesen. In den Jahren nach Nickischs verdienstvollem Metzler-Band hat die Briefforschung eine medientheoretische Wende genommen: am Anfang stand Siegerts extravagante Geschichte der Literatur als einer Geschich-

\footnotetext{
6 Steinhausen 1889, III.

7 Steinhausen 1889, III.

8 Steinhausen 1891, 245.

9 Nickisch 1991, 29-92.

10 Vgl. Nickisch 1991, 44.

11 Nickisch 1991, 44.

12 Nickisch 1991, 44.
} 
te ihrer „Geschicke“ in der „Epoche der Post“;"13 besondere Beachtung fand Albrecht Koschorkes „Mediologie des 18. Jahrhunderts“, die es in Form eines kultursemiotischen close readings unternahm, die Topoi der Briefkultur und die Verhandlungen des Körpers zueinander in ein Verhältnis zu setzen und ihre „innere Stimmigkeit“ nachzuzeichnen. ${ }^{14}$

Jenseits von Historismus, Sozialgeschichte und Kultursemiotik bekennt sich der vorliegende Sammelband zu einer Geschichtsschreibung des Briefs im Zeichen einer Repragmatisierung. ${ }^{15} \mathrm{Er}$ ist zwar weit davon entfernt, die Lücke zu schließen, auf die Albrecht Schöne aufmerksam gemacht hat, aber er möchte einleitend doch der Frage nachgehen, wie eine solche Geschichte des Briefs aussehen könnte, und er möchte anhand einzelner Fallstudien die Geschichtlichkeit des Briefs erkunden. Dazu ist es zunächst nötig, zu klären, von welchem Phänomen hier die Rede ist.

\section{Ereignis und Objekt - begriffliche Differenzierungen}

Einschlägige Lexikonartikel behandeln den Brief wie eine literarische Gattung oder ein rhetorisches Mittel: Sie stellen überzeitliche, mehr oder weniger verbindliche Charakteristika des Briefes neben eine Geschichte des Phänomens, die auf einer Reihe herausragender, editorisch erschlossener Beispiele beruht. Je nach Ausrichtung des Lexikons erhält das Chamäleon Brief dabei ein anderes Gesicht.

Der Neue Pauly behilft sich bei einem Minimum an Normativität - in der Antike war Brieftheorie die Ausnahme - auf nur vier Spalten mit einer an Gebrauchsfunktion und ästhetischem Anspruch orientierten Typologie (Briefsortenlehre), einer am Kommunikationsbegriff festgemachten Definition, wenigen Informationen zu Materialität und Übertragungsfunktion und schließlich mit Notizen zur „Geschichte des Briefeschreibens“, die lediglich wichtigste Überlieferungsstränge und wiederum historisch wechselnde Funktionen benennen. ${ }^{16} \mathrm{Zu}$ weit voneinander entfernt sind diese Überlieferungsstränge, als dass ,eine“ Geschichte des antiken Briefs geschrieben werden könnte, vielleicht mit Ausnahme der Beobachtung, dass

13 Vgl. Siegert 1993; für eine frühe, mediengeschichtlich argumentierende Skizze zur Geschichte des Briefes „im Zeitalter der technischen Reproduzierbarkeit der Schrift“ vgl. Vellusig 1991.

14 Koschorke 1999, 104. Dass Koschorkes „Mediologie“ auch auf Widerspruch gestoßen ist, steht auf einem anderen Blatt. Vgl. statt anderer Stiening 2001.

15 Vgl. die Plädoyers zugunsten einer pragmatischen Geschichtsschreibung bei Tschiggerl et al. 2019, 11 und Buschmeier 2014.

16 Schmidt u. Neumann 1997. 
das Formular aus Anrede, Eingangs- und Abschiedsgruß sich von einfachen zu komplexeren Formen entwickelt habe. Beobachtungen von Varianz und Invarianz stehen also nebeneinander, ohne zu einem Bedingungsgefüge zu finden.

Ähnlich verfährt das Historische Wörterbuch der Rhetorik, auch wenn es nicht mehr rein deskriptiv vorgehen kann, insofern rhetorische Normen des Briefs aus systematisch-theoretischen Quellen erschlossen werden. ${ }^{17}$ Doch liegt der Schwerpunkt des Artikels auf dem Referat einer Geschichte des Briefs bis ins achtzehnte Jahrhundert hinein. Unschärfen schleichen sich aufgrund der Vorannahme einer Nähe des Briefs zur Literatur ein, die zugleich historisch-evolutionäre Postulate konterkariert, denn der Brief sei ,eine literarische Gattung, die in der Antike schon in der gesamten Vielfalt ihrer Erscheinungsformen vertreten ist" “. ${ }^{18}$ Die folgenden Abschnitte, die den Privatbrief als Highlight der Briefgeschichte darstellen und den literarisierten Brief als dessen Gegenpol beschreiben, sehen von einer Befragung rhetorischer Inventare zugunsten einer Kontamination von Gattungs- und Epochengesichtspunkten im Grunde ab.

Die jüngere Briefforschung hat gegen diese spezifisch literaturwissenschaftliche Fixierung auf die Textualität ihres Gegenstands Einspruch erhoben und auf die Medialität und die Materialität des Briefes aufmerksam gemacht. „Sobald Briefe Texte sind“, so das pointierte Diktum von Anne Bohnenkamp und Waltraud Wiethölter, „sind Briefe keine Briefe mehr“. ${ }^{19}$ Die Beiträge des Bandes schließen sich dieser Perspektive an, ohne doch die Textualität des Briefes aus den Augen zu lassen oder Gattungsfragen zu ignorieren. Das erfordert begriffliche Differenzierungen. Der Brief soll hier zunächst als Kommunikationsmedium verstanden werden und sowohl vom Brief als Kommunikationsform als auch vom Brief als einer literarischen Gattung unterschieden werden, wobei diese Bestimmungen keine Alternativen darstellen, sondern in einem inneren Bedingungszusammenhang stehen. $^{20}$

Kommunikationsmedien sind „konkrete materielle Hilfsmittel, mit denen Zeichen verstärkt, hergestellt, gespeichert und/oder übertragen werden können“.21 Betrachtet man den Brief als Kommunikationsmedium, so ist er als schriftlich codierte Mitteilung zu bestimmen, die im Regelfall verschlossen versandt und individuell

\section{Vgl. Müller 1994.}

18 Müller 1994, 66. Der Autor des Artikels zeichnet denn auch für einen Brief-Artikel in einem Handbuch literarischer Gattungen verantwortlich, der den literaturwissenschaftlichen Horizont der Briefforschung nicht verleugnet und eine Briefgeschichte als literarische „Gattungsgeschichte“ (Müller 2009, 80) erzählt.

19 Bohnenkamp u. Wiethölter 2008, IX.

20 Zum Folgenden vgl. Vellusig 2018, 57-61.

21 Holly 1997, 69-70. 
(per Bote) oder auf dem anonymen Postweg übermittelt wird. Die Medialität des Briefes begründet zugleich seine (inzwischen gut erforschte) Materialität ${ }^{22}$ : den Status des Briefes, ein Objekt zu sein, das sich über den medialen Vermittlungsprozess hinaus erhalten hat; und sie begründet die Möglichkeit, das Objekt, das seine primäre Funktion erfüllt hat, aufzubewahren.

Briefe sind keine Bücher, weder geschriebene noch weniger gedruckte. Das Manuskript eines Buches zielt auf seine „Selbstaufhebung in der Typographie“; die Handschrift des Briefes ist dessen „Echtheitssiegel““.23 Werden Briefe gedruckt, dann unterliegen sie einem fundamentalen Wandel: Sie wechseln ihren medialen Status und werden zu Texten, die von Menschen gelesen werden, für die sie nicht geschrieben wurden. Aus medialer Perspektive betrachtet, haben gedruckte Briefe aufgehört, Briefe zu sein - sie treten in ihre Nachgeschichte ${ }^{24}$ ein. Zu dieser medialen Nachgeschichte des Briefes gehört auch das Sammeln von Briefen als Unikaten und individuellen Lebenszeugnissen. Seit etwa 1800 bildete sich in Europa eine regelrechte Sammlerszene, die der Beitrag von Claudia Bamberg anschaulich nachzeichnet und bis ins frühe zwanzigste Jahrhundert verfolgt. Sie war weniger an Texten als an der Aura des einmaligen Gegenstandes interessiert, der die als physiognomisch begriffene Handschrift des Schreibers trug. ${ }^{25}$

Als Kommunikationsform ist der Brief durch die medialen Rahmenbedingungen interaktionsfreier, individuell adressierter Kommunikation geprägt, aber er ist dadurch weder thematisch noch funktional festgelegt. ${ }^{26}$ Man kann in Briefen über alles Mögliche schreiben, man kann damit alle möglichen Zwecke verfolgen, man kann es mehr oder weniger formell oder formbewusst tun - aber man muss sich schriftlicher, jedenfalls graphischer Mittel bedienen, und man muss ein Gegenüber adressieren: eine konkrete Person oder einen intimen Zirkel, im historisch spezifizierten Fall eine Glaubensgemeinschaft. Anders als das Speichermedium Buch ist das Kommunikationsmedium Brief kein Broadcast-, sondern ein Punktzu-Punkt-Medium: „Während sich beim Buchdruck der Adressat selbst bestimmt, bestimmt der Brief seinen Adressaten selbst.“27

22 Vgl. statt anderer Bohnenkamp u. Wiethölter 2008; Henzel 2020; am Beispiel Fontanes: Gabler 2020.

23 Müller 2012, 161. Digitale Editionen wirken diesem Statuswechsel entgegen, insofern sie den Objektcharakter des Briefs bewusst halten.

24 Rainer Baasner hat in diesem Sinne zwischen einer ,primären“ („Briefkultur I“) und einer ,sekundären“ Briefkultur („Briefkultur II“) unterschieden. Vgl. Baasner 1999, 14 u. $27-29$.

25 Vgl. Heumann 2020.

26 Vgl. Dürscheid 2003, 40.

27 Bohn 1997, 44. 
Kommunikation im Medium des Briefes ist - wie schriftgestützte Kommunikation generell - ,zerdehnt ‘. ${ }^{28}$ Zwar ist der Brief durch Merkmale wie Anrede-, Grußund Abschiedsformeln explizit als funktionales Äquivalent einer zwischenmenschlichen Begegnung ausgewiesen (die Etablierung eines „idealisierten Dialograums“ durch spezifische „Eröffnungs“- und „Abschlusshandlungen“ gehört zu den „Basisregeln“ der Kommunikation), ${ }^{29}$ aber das Schreiben ist nicht an das Hier und Jetzt einer gemeinsamen Kommunikationssituation gebunden. Auch wenn ein Brief an den anderen anknüpfen mag, ist briefliche Kommunikation monologisch strukturiert. Das verleiht dem Geschriebenen textuelle Qualitäten: Texte sind nach Konrad Ehlich „durch ihre sprechsituationsüberdauernde Stabilität gekennzeichnet“. ${ }^{30}$ Briefe überdauern ihre Ereignishaftigkeit nicht nur als bloße Objekte, sondern vor allem auch deshalb, weil sie in ihrem Wortlaut auf Überlieferung bezogen sind.

Als Textsorte (oder Gattung) im engeren und eigentlichen Sinn lässt sich der Brief nur dann ansprechen, wenn die Anlässe und Beweggründe des Schreibens so gleichförmig sind, dass sich dabei spezifische Muster ausbilden. Textsorten oder allgemeiner gesprochen: ,kommunikative Gattungen“ sind Routinen, die mehr oder weniger verpflichtende Lösungen für bestimmte kommunikative Probleme bereitstellen. ${ }^{31}$

Solche Routinen, Glück zu wünschen, sein Mitgefühl zu bekunden, eine Bitte vorzubringen, sich um eine Stelle zu bewerben, eine Empfehlung auszusprechen usw., gehörten Jahrhunderte lang eben deshalb zum Standardrepertoire von Briefstellern, weil mustergültige Lösungen für Formulierungsprobleme den einzelnen Briefschreiber von der Mühsal des Formulierens entlasten. - Anders verhält es sich mit Briefen, die thematisch wie funktional nur wenig oder gar nicht festgelegt sind, mit solchen Briefen also, die sich allenfalls dadurch charakterisieren lassen, dass sie zwischenmenschliche Beziehungen gestalten und kultivieren. Zwar bilden auch sie Routinen aus, aber diese Routinen lassen sich nicht in derselben Weise $\mathrm{zu}$ verpflichtenden Lösungen von Kommunikationsproblemen verdichten - vielmehr bewegt sich der Brief in diesem Falle, nicht anders als die mündliche Kommunikation, in einem unabsehbaren Spektrum von Möglichkeiten.

Wenn die Beiträge dieses Sammelbands die Geschichtlichkeit des Briefes erkunden, dann haben sie primär die Kommunikationsform Brief und den Reichtum ihrer textuellen Formate im Blick. Diese Ausweitung der Perspektive gegenüber einer gattungsgeschichtlichen Betrachtungsweise erlaubt es, sowohl den Wandel

28 Vgl. Ehlich 1993, 32; Peter Bürgel hat vom „Phasenverzug“ (Bürgel 1976, 288) brieflicher Kommunikation gesprochen.

29 Vgl. Kilian 2005, 129.

30 Ehlich 1993, 32.

31 Vgl. Luckmann 1988, 283. 
dieser Kommunikationsform Brief als auch ihre bemerkenswerte Kontinuität in den Blick zu nehmen.

\section{Anfänge}

Die frühesten datierbaren Briefe sind dem dritten Jahrtausend vor Christus zuzuordnen, die keilschriftliche Briefüberlieferung aus den Reichen der Sumerer und der Akkader beginnt um 2370. Wer die Texte dieser mehr oder weniger fragmentarisch erhaltenen Briefe in Übersetzung liest, wird bis heute charakteristische Merkmale wiedererkennen: die Nennung von Absender und abwesendem Adressaten; neben dem Formelhaften mancher Passagen (vor allem der Einleitung) stehen Mitteilungen und Aufforderungen. Oft regeln die Herrschenden den Handel oder zu leistende Abgaben, arrangieren Eheschließungen. ${ }^{32}$

Ein auf einer Tontafel in akkadischer Sprache erhaltener Brief aus dem dreiundzwanzigsten Jahrhundert, der als einem Transport zugeführter Gegenstand bis heute daran kenntlich ist, dass die Tafel durchbohrt war, also wohl vom Boten oder der Empfängerin um den Hals getragen wurde, kommt unserer heutigen Erwartungshaltung von einer persönlichen Note und bestenfalls dem Unikalen in Aussage und Stil vielleicht schon nahe. Auch die thematische und kommunikative Variabilität des Briefs, die oft harte Fügungen zur Folge hat, ist bereits erkennbar: „Folgendermaßen Babi zu Šārtum: Da kann ich, wie man so sagt, nur lachen! Warum streitet ihr; du und Ibbi-ilum, im Hause? Lebt (in Eintracht) zusammen! Schicke mir ,Baumöl'‘"“33

Hierbei handelt es sich um Einzelfunde. Die knapp 400 Tontafeln, die im späten neunzehnten Jahrhundert in Ägypten, dem heutigen Tell el Amarna, gefunden wurden, entstammen dem Palastarchiv des Pharaos Echnaton in seiner Residenzstadt Achet-Aton. Sie datieren vor allem aus dem vierzehnten Jahrhundert. Als archiviertes Ganzes wurden diese Briefe von vornherein wahrgenommen, wenngleich viele teilweise zerstört, ja kaum lesbar waren und Selektionskriterien $\mathrm{zu}$ ermitteln und damit eine Erklärung dafür $\mathrm{zu}$ finden, dass ausgerechnet jenes Korpus ,überlebt' hatte, schwer fiel. ${ }^{34}$ Ein gemeinsamer Nenner ergab sich allerdings rasch: die Kommunikation zwischen Ägypten und den Mächten des Zweistromlandes, Vasallen einerseits, als ebenbürtig betrachtete Partner andererseits.

32 Vgl. Neumann 2006.

33 Neumann 2006, 10.

34 Vgl. Moran 1992; Schniedewind 2015. 
Die Briefe enthalten Forderungen und Anordnungen des Pharaos und umgekehrt Rückmeldungen von Vasallen, sie begleiten die Sendung eines Gegenstandes.

Forschung ist hier gleichsam Erklärung des überlieferten Befundes. Soll der Brief aber zum privilegierten Gegenstand einer Untersuchung werden, gilt es, die Forschungsfrage zu präzisieren, die dabei verfolgt wird. In diesem Sinne hat der Altorientalist Walther Sallaberger Interaktion und Textgestaltung in altbabylonischen Alltagsbriefen aus der Zeit Hammurabis von Babylon und seiner Nachfolger untersucht. Sallaberger definiert Briefe anhand der durch sie realisierten sprachlichen Interaktion mit räumlich entfernten Adressaten und spricht, wie das auch im vorliegenden Sammelband der Fall ist, von einer „Kommunikationsform“. ${ }^{35}$ Neben stereotypen Formeln finden sich eine große inhaltliche Bandbreite und eine enorme Vielfalt an Formulierungsoptionen. Erkennbar ist damals schon der später von der Rhetorik geforderte Aufbau in salutatio - narratio - petitio. ${ }^{36}$ Modern mutet an, dass bei aller Formelhaftigkeit emotionale Bindungen brieflich konkretisiert werden:

Einen Gruß von Dir hast Du selbst nie geschrieben. Nie hörte ich einen Ton von Dir, so daß es mir zutiefst schlecht geht. Man erzählt mir von Deiner ärgerlichen Lage, so daß ich täglich um Dich weine. Und B., warum ist sie besorgt? Schreibe einen Gruß von Euch, damit ich nicht mehr weinen muß. Euer Heil [...] täglich [...].37

Diese Beispiele verweisen zurück auf die älteste Überlieferung von Briefen und sie liebäugeln mit einer doppelten Perspektive: Einerseits konstituieren sich Briefe von Anfang an klar und deutlich aufgrund ihrer Textualität - sobald ein beschriebenes Objekt oder ein gedruckter Text bestimmte Merkmale besitzt, identifizieren wir ihn als Brief. ${ }^{38}$ Andererseits können wir schon in dieser Frühzeit eine Art von evolutionärem Komplexitätszuwachs ausmachen. Sein Nachweis ist allerdings erst bei dichter werdender Überlieferung möglich.

Hiervon profitiert der Althistoriker Patrick Reinard, der in seiner materialund umfangreichen Untersuchung des Brief- und Warenverkehrs im ersten bis dritten Jahrhundert nach Christus im kaiserzeitlichen Ägypten zu dem Ergebnis kommt, dass private Papyrusbriefe Kommunikationsnetzwerke schufen, deren Ziel es war, Transaktionskosten von Waren zu senken. Das Briefeschreiben war der Ökonomie (der des Warentauschs und der des Schreibens selbst) unterworfen,

35 Sallaberger 1999, 8. - Sallaberger übernimmt den Begriff von Reinhard Nickisch, der damit die Gemeinsamkeiten mit der mündlichen Kommunikation hervorhebt (vgl. Nickisch 1991, 9).

36 Vgl. Sallaberger 1999, 143. Die von der rhetorischen Brieflehre empfohlene captatio benevolentiae und eine conclusio (vgl. Müller 1994, 62) fehlen allerdings noch.

37 Zit. nach Sallaberger 1999, 102.

38 Vgl. dazu den Beitrag von Sophia Wege in diesem Band. 
dennoch eröffnet sich bei Lektüre dieser Papyri ein Panorama des Alltagslebens der Zeit. ${ }^{39}$ Zeitlos beinahe wollte man den Brief eines gekränkten Jungen namens Theon nennen, der im zweiten oder dritten Jahrhundert seinem Vater schreibt, der das Versprechen, ihn mit auf eine Reise nach Alexandria zu nehmen, nicht gehalten hatte:

\footnotetext{
Das hast Du schön gemacht, nicht mitgenommen hast Du mich mit Dir in die Stadt. [...] Wenn Du mich nicht mit Dir nach Alexandria nehmen willst, dann werde ich Dich (sic) weder einen Brief schreiben noch spreche ich mit Dich (sic), noch wünsche ich Dich (sic) Gesundheit. Wenn Du nach Alexandria gehst, nehme ich keine Hand von Dir und grüße Dich nie wieder. Wenn Du mich nicht mitnehmen willst, wird es so. [...] Wenn Du nicht (nach mir) schickst, esse und trinke ich nicht. So steht es! ${ }^{40}$
}

Ist es also ein Irrtum anzunehmen, der Brief besitze eine spezifische ,Modernität‘, die ihn im achtzehnten und bis ins neunzehnte Jahrhundert hinein in Europa zum Gegenstand literarischer Gestaltungsbemühungen werden ließ? Ist er nicht immer schon das thematisch flexible, leicht gebrauchsfertige Alltagsobjekt, das dem zeitlich zerdehnten schriftlichen Austausch zwischen räumlich getrennten Personen dient? Die babylonischen Alltagsbriefe des achtzehnten und siebzehnten Jahrhunderts vor Christus dienen, so der ebenso schlichte wie unabweisbare Befund Walther Sallabergers, „der direkten Kommunikation zwischen konkreten Individuen". ${ }^{41}$

\section{Distanzkommunikation und Distanzregulation}

Die Geschichte eines kulturellen Phänomens lässt sich nur schreiben, wenn man dieses Phänomen als etwas identifiziert, das als solches gleichbleibt, während es sich wandelt. In der Forschung werden die bemerkenswerte Kontinuität des Briefs und damit auch der Wandel seiner Formate primär kommunikationstheoretisch begründet. Reinhard Nickisch hat die Geschichte des Briefes auf die „Grundfunktionen der Informationsübermittlung, des Appellierens und der Selbst-Äußerung““2 zurückgeführt: Diese machen „die genuinen und historisch quasi-invarianten kommunikativen Möglichkeiten des Briefes“ aus; ihr Vorherrschen kann „für einzelne

39 Vgl. Reinard 2016, I.

40 Vgl. Reinard 2016, I, 15.

41 Sallaberger 1999, 12.

42 Nickisch 1991, 13. Ähnlich dann Dücker 1992, 124; Guntermann 1997, 54. 
Phasen oder Epochen der Briefgeschichte“ signifikant sein. ${ }^{43}$ In diesem Sinne hat Johannes Anderegg den Strukturwandel der Briefkultur im achtzehnten Jahrhundert als Akzentverschiebung von einem dominant appellierenden zu einem dominant ausdruckshaften Schreiben gedeutet. ${ }^{44}$

Man kann das noch grundlegender fassen und - wie Sophia Wege dies in ihrem evolutionsbiologisch argumentierenden Beitrag zum vorliegenden Band tut - zwischen dem „Inhalts-“ und dem „Beziehungsaspekt“ (Watzlawick) zwischenmenschlicher Kommunikation unterscheiden. Briefe dienen dem Austausch von Informationen und sie gestalten zwischenmenschliche Beziehungen. Solche Überlegungen rücken basale Bestimmungen der Kommunikation in den Blick und sie legen es nahe, diese Bedingungen brieflicher Kommunikation ihrerseits anthropologisch zu fundieren und so Gesichtspunkte zu gewinnen, die es erlauben, Fragen nach der Textualität des Briefs mediengeschichtlich zu kontextualisieren.

Robert Vellusig hat in einzelnen Beiträgen vorgeschlagen, die Kontinuität und den Wandel der Kommunikationsform Brief in diesem Sinne auf eine Kontinuität menschlicher Bedürfnisse zurückzuführen, die in je spezifischen kulturellen Rahmenbedingungen je spezifische Ausprägungen findet. ${ }^{45}$ Die Merkmale der Schriftlichkeit und Materialität, der Adressiertheit und des Transports charakterisieren den Brief als Medium schriftlicher Distanzkommunikation. Sie weisen ihn als Mittel aus, eine soziale Distanz zu überbrücken. Die Distanz zwischen zwei Menschen ist aber nicht nur ein räumliches, sondern auch ein psychisches Phänomen; sie lässt sich nicht nur physisch, sondern auch symbolisch regulieren. Räumliche und symbolische Distanzregulation sind Mittel der Wahl, zu Artgenossen eine optimale Distanz einzunehmen bzw. eine nicht optimale Distanz zu kompensieren - jenen nahe zu sein, die einem ,nahe stehen', jenen aus dem Weg zu gehen, denen man ,nicht über den Weg traut', sich jenen zu ,öffnen', deren Nähe man sucht, sich gegenüber jenen ,bedeckt zu halten', denen man misstraut und die man ,nicht an sich heranlassen' möchte.

Anders als physische Distanz, die von beiden Seiten aus gleich groß ist, ist psychische Distanz eine asymmetrische Relation. Wie die Metaphorik zeigt, lässt sich ihre symbolische Regulation daher angemessener als Befestigung oder Verwischung einer Grenze fassen. In der Face-to-Face-Interaktion geschieht das auf vielfältige, nicht vorrangig verbale Weise: durch Gesten der Zu- und Abwendung, durch den Blickkontakt und die Art der Stimmführung, durch die mehr oder we-

43 Nickisch 1991, 13.

44 Vgl. Anderegg 2000, 15.

45 Zum Folgenden vgl. Vellusig 2011, 2018, 2020, 2021. Das Konzept der „sozialen Distanz“ wurde von dem Psychologen Norbert Bischof entwickelt. Grundlegend: Bischof 1993; vgl. auch Bischof 2008, 401-402. 
niger vertraulichen Formen der Anrede und die Intimität der Gesprächsthemen, durch die plastischen und drastischen Ausdruckmittel expressiver Mündlichkeit, die etwas davon verraten, wie und wonach jemandem zumute ist.

Mit dem Konzept der symbolischen Distanzregulation ist also nicht nur die Medialität des Briefes erfasst (die Tatsache, dass er eine räumliche Distanz überwindet), sondern auch seine Textualität, $d$. h. sein von der modernen Briefforschung besonders betontes „inszenatorisch-fiktionales Potential“. ${ }^{46}$ Briefe stellen zwischenmenschliche Nähe mithilfe symbolischer Distanzäquivalente her: „Wie viel Nähe oder Distanz in einem Schreiben gewünscht wird“, so Rainer Baasner, ,bleibt von der räumlichen Entfernung unabhängig. “ ${ }^{47}$ Nähe und Distanz sind kommunikative Ausdrucksgestalten. Sie beruhen auf verbalen Strategien, mit denen sich jemand als Person zur Geltung bringt oder sich verbirgt und die es dem anderen erleichtern oder erschweren, mit ihm in Beziehung zu treten. Im Brief geschieht das beinahe ausschließlich auf verbale Weise. Was sich im zwischenmenschlichen Umgang von selbst ergibt, muss hier bewusst gestaltet werden. Während es in der interpersonalen Dynamik der Interaktion einer besonderen Anstrengung bedarf, sich zu verbergen, gehört es zu den Herausforderungen des Schreibens, als Person überhaupt sichtbar zu werden. Immer dort, wo das Schreiben von Briefen als Möglichkeit kultiviert wird, Beziehungen zu pflegen und sich als Person zur Geltung zu bringen, wird Selbstdarstellung zur literarischen Herausforderung und zum kommunikativen Problem.

Einige Beiträge des Bandes zeigen das auf besonders anschauliche Weise: Isabelle Stauffer anhand der galanten Briefkultur rund um Aurora von Königsmarck, die von der Sorge bewegt ist, dass das literarische Rollenspiel als Wirklichkeit ernstgenommen werden und die beteiligten Personen diskreditieren könnte; Gideon Stiening anhand eines spektakulären Betrugsfalles rund um den Historiker Johannes von Müller, der zum Opfer eines jungen Mannes wird, der ihm als vermeintlicher Graf Louis Batthyány Szent-Iványi Liebesbriefe schreibt und der als Postillon d'Amour diese Briefe mit ihm liest - dem sehnsüchtig liebenden Müller zum Greifen nah und zugleich doch auch ungreifbar fern.

Die Frage nach der rechten Distanz gehört seit je zu den zentralen Fragen der Epistolographie. Sie wird zunächst durch die Lehre von der Stilhöhe, vor allem aber durch das ausgesprochen differenzierte Spektrum der Titel und Anredeformen geregelt; und sie bleibt, wie einige Beiträge des Bandes dokumentieren, auch

46 Vgl. Schuster u. Strobel 2013, XIII.

47 Baasner 1999, 2. Dass die monologische Verfasstheit des Briefs und die Sehnsucht nach Nähe die übliche Länge von Briefen erheblich erweitern kann, zeigt die exemplarische Fallstudie von Ulrich Breuer und Anke Lindemann in diesem Band. Die Länge eines Briefes ist ein Indiz für Vertrautheit und zeichnet den Brief als besondere Gabe aus. 
nach der Auflösung der rhetorischen Stillehre, virulent: Rotraut Fischer zeichnet das am Beispiel des Briefwechsels von Christian Bang und Friedrich Carl von Savigny nach; Olivia Varwig zieht die Linien bis in die Gegenwart aus und macht anschaulich, dass sich die angemessene Nähe zum Kommunikationspartner auch räumlich zeigt: sie materialisiert sich im Spatium. Einem besonderen Fall der Vergegenwärtigung der Person im Medium des Briefes ist der Beitrag von Eve-Marie Becker gewidmet: Er porträtiert das literarische self fashioning Senecas und Paulus' - ihre mit souveräner oder auch autoritativer Geste vollzogene Selbstpräsentation als Lehrer und Apostel.

Briefe sind individuell adressierte Texte. Die Exklusivität der Adressierung zieht um die Kommunikationspartner eine Grenze, die den idealisierten Dialograum des Briefs vor der Zudringlichkeit Dritter schützt. Die Verhüllung der Botschaft (durch Umschlag, Siegel u. ä.) macht sinnfällig, dass hier eine individuelle Beziehung hergestellt wird, von der andere ausgeschlossen sind; im Briefgeheimnis wird diese kommunikative Grenze gleichsam als Berührungsverbot institutionalisiert. Das gilt auch für die Briefe, die - wie im Falle der Paulus-Briefe - an eine Gemeinschaft Gleichgesinnter gerichtet und - nicht anders als die Briefe Senecas gemeinschaftsbildend sind. Jochen Strobel macht in seinem Beitrag mit Nachdruck auf die Netzwerkaffinität des Briefs aufmerksam: Ein Brief kommt selten allein und er findet nicht selten mehrere Leserinnen und Leser. Aus Briefen entstehen kommunikative Infrastrukturen zwischen mehreren Personen, soziale Korrespondenznetzwerke, an denen nicht nur diejenigen beteiligt sind, die Briefe schreiben und empfangen, sondern auch all jene, die Briefe mitlesen oder in Briefen gegrüßt werden. Auch diese Netzwerke etablieren Grenzen, nicht zuletzt im Aufbau einer wissenschaftlichen Community und ihres internen Wissenstransfers. ${ }^{48}$

\section{Geschichte und Geschichtlichkeit des Briefs}

Man wird, wenn man diese Praktiken der sozialen Distanzregulation verfolgt, vielleicht auf shifting baselines stoßen, auf die schleichende Verschiebung der intuitiven Grenzen zwischen Intimität, Vertrautheit und Distanz (nicht zuletzt

48 Beispielhaft wird dies kenntlich anhand der Begründung der europäischen ,Indologie‘, die über internationalen Austausch, Archivbesuche, Zeitschriftengründungen und natürlich Editionen läuft - aber auch noch ganz wesentlich über Korrespondenzen, wovon die Briefe, die die deutsche Gründerfigur August Wilhelm Schlegel tauschte, nur einen Ausschnitt bilden. Vgl. Hanneder 2016; Strobel 2018. 
zwischen den Generationen und Geschlechtern), ${ }^{49}$ wie sie sich besonders prägnant an der Geschichte der Anredeformen ablesen lässt. In dieser Hinsicht wäre eine Kulturgeschichte des Briefs Teil einer umfassenderen Kulturgeschichte der Umgangsformen, d. h. der Grenzen, die Menschen in ihrem Ausdrucksverhalten wahren, und der Werte, an denen sie sich dabei orientieren: einer Kulturgeschichte der Diskretion und des Taktgefühls, der Höflichkeit und des Respekts (Rüdiger Görner hat von einer „Ethik des Briefeschreibens“50 gesprochen), aber auch des Maskenspiels und Humors. - Die Beiträge von Isabelle Stauffer, Jana Kittelmann und Eva Lia Wyss lassen sich als Beiträge zu einer Geschichte medial vermittelter Vertrautheit lesen und vermitteln eine anschauliche Vorstellung davon, dass solche Grenzverschiebungen tatsächlich stattgefunden haben.

Die Modulation von Nähe und Distanz ist nicht nur eine Frage von Konventionen, sondern auch von ästhetischen Standards, die sich in einer Kultur etabliert haben. Sie provoziert die Frage nach dem Verhältnis, in dem eine Kulturgeschichte des Briefes zur Geschichte der literarisch-literalen Kultur als ganzer steht. Weil es dabei um Artikulationsstandards des literarischen Schreibens geht, ist die Literaturwissenschaft tatsächlich auf besondere Weise berufen, sich der Geschichte des Briefes anzunehmen; aber es wäre doch verfehlt, diese Geschichte in eine einfache Analogie zur Geschichte der Literatur und ihrer Epochen zu setzen. Das hat mit dem medialen und kulturellen Status des Briefs zu tun: Zwar kennt auch die Geschichte des Briefes kulturelle Lernprozesse: Formen der Traditionsbildung, die auf Nachahmung und Unterricht beruhen; der Brief bildet aber nicht mit derselben Zwangsläufigkeit einen der Geschichte der Literatur vergleichbaren Traditionszusammenhang aus. Er war und ist zunächst einfach nur eine Form des schriftlichen Kommunizierens. Der Verfasser eines Briefes ist keinem state of the art verpflichtet und auf keinen Verleger angewiesen, der seinen Text einer anonymen Medienöffentlichkeit vermittelt. Über das Gelingen brieflicher Kommunikation entscheiden allein diejenigen, die auf exklusive Weise an ihr beteiligt sind.

Michel Foucault hat deshalb zu Recht darauf hingewiesen, dass Briefe zwar einen Verfasser (einen „Schreiber“), aber keinen Autor haben. ${ }^{51}$ Die Kategorie „Autorschaft" ist an das Buch bzw. das Medium Buchdruck gebunden, insofern erst durch die Publikation von Schriften, d. h. durch ihre Adressierung an ein anonymes Lesepublikum, Öffentlichkeit konstituiert wird. ${ }^{52}$ Begründet ein Brief oder eine Folge von Briefen also einerseits keine Autorschaft, so zählen Briefe zum Werk

49 Vgl. Bauer u. Hämmerle 2017.

50 Görner 2008, 21. Dass diese Ethik des Briefschreibens auch die Qualität des Briefes als einer Denkform begründet, macht Görners Beitrag zu diesem Sammelband deutlich.

51 Foucault 1974, 17.

52 Vgl. Chartier 1992, 59. 
eines Autors andererseits doch hinzu. Die Probe aufs Exempel sind die Briefe der ,Romantiker“, denen ,romantische“ Qualitäten wie reizvolle „Bruchstückhaftigkeit“ (Gundolf) zugeschrieben wurden - oder im Einzelfall die Begründung ,ästhetischer Subjektivität“ (Bohrer). ${ }^{53}$ Für die literarische Moderne mögen stellvertretend die Namen Hofmannsthal und Rilke stehen. ${ }^{54}$ - Dass sich Autoren wie Rilke oder Jünger in ihren Briefen immer auch als Autoren profilieren und Werkpolitik betreiben, machen die Beiträge von Alexander Honold und Norman Kasper deutlich: Während sich Rilkes Korrespondenz mit seinem Verleger Kippenberg unter die Vorzeichen einer ambivalenten Modernität setzen lässt, die den inspirationsästhetischen Anspruch mit einer pragmatischen Orientierung an Buchwesen und literarischer Öffentlichkeit zu versöhnen sucht, erweisen sich Jüngers Briefwechsel mit Naturwissenschaftlern als strategische Knotenpunkte im Netz der Begründung einer epistemischen Autorschaft. ${ }^{55}$

Das innovative Potential, das der Brief für die Literatur des achtzehnten Jahrhunderts besaß, beruhte darauf, dass die Schrift mit ihm den kommunikativen Alltag erreichte und zur Verschriftlichung von Ausdrucksformen führte, die bislang außerhalb der durch Latinität und Rhetorik geprägten, exklusiv männlichen Schriftkultur lagen. ${ }^{56}$ Der Briefroman des achtzehnten Jahrhunderts war dessen unmittelbarer Reflex. (Der Beitrag von Yulia Mevissen erinnert daran und spinnt den Faden in die Gegenwart fort.) - Auch wenn es also tatsächlich einen engen Zusammenhang zwischen Briefkultur und Literatur gab, auch wenn die Forschung herausragende Briefe kennt und einzelne Briefe wie ,Goethes Brief an Behrisch ${ }^{57}$ oder Kafkas ,Gespensterbrief‘ aus der Korrespondenz mit Milena Jesenská inzwischen den Status von Klassikern gewonnen haben - wer immer sich anschickt, einen Brief zu schreiben, kann das tun, ohne sich in Brief-Anthologien umgetan zu haben oder überhaupt ein Liebhaber der Literatur zu sein, geschweige denn: literarische Interessen zu verfolgen. Dem je individuellen Reiz solcher Briefe tut dies keinen Abbruch. Das gilt für einen schreibenden Goldschmiedegesellen ${ }^{58}$ so gut wie für den der deutschen Schriftsprache kaum mächtigen Kronprinzen

53 Vgl. Gundelfinger 1907; Bohrer 1987.

54 Vgl. Košenina 2002; Schuster 2014; Honold et al. 2019.

55 Die Konzentration auf autor- und werkpolitische Fragen führt von den ,eigentlichen‘ Texten keineswegs weg, im Gegenteil: Der Fokus auf jene kommunikativen Strategien, die sich ganz maßgeblich dem medialen und (archiv-)kulturellen Status der Briefe verdanken, ermöglicht neue Blicke auf die Entstehung von Rilkes Aufzeichnungen des Malte Laurids Brigge oder die Duineser Elegien bzw. auf Jüngers essayistische Prosa und fiktionale Texte der 1950er Jahre.

56 Vgl. Ter-Nedden 1988, 173; Vellusig 2011, 159-162.

57 Die Kanonisierung dieses Briefes begründet hat die Studie von Schöne 1967; vgl. auch Schöne 2015, 73-122.

58 Vgl. Ulbricht 2009. 
von Preußen. ${ }^{59}$ Deshalb hat die von Reinhart Koselleck geprägte Formel von der „Geschichte“ als der „Gleichzeitigkeit von Ungleichzeitigem“ bzw. der „Ungleichzeitigkeit von Gleichzeitigem" 60 für die Geschichte des Briefs eine besondere Relevanz. Man kann sich Briefe auch dann schreiben, wenn man an der literarisch-literalen Kultur selbst nicht oder allenfalls rudimentär partizipiert. Die von Jens Ebert erforschten, edierten und im vorliegenden Band schlaglichtartig charakterisierten Briefe, die die Soldaten der beiden Weltkriege und ihre Angehörigen einander schreiben, sind Lebenszeugnisse existenzieller Not; sie stehen in keinem epistolaren Traditionszusammenhang, sondern entstehen aus dem elementaren Bedürfnis, einander Nachricht zu geben und einander schreibend nah zu sein - ganz unabhängig davon, welcher Grad an Nähe im Duktus des Schreibens Gestalt gewinnt.

Ein Begriff von Geschichte, der mit der "Gleichzeitigkeit des Ungleichzeitigen“ rechnet, setzt - wie Koselleck deutlich gemacht hat - Fortschrittserfahrungen voraus. ${ }^{61} \mathrm{Ihn}$ zu verwenden, impliziert, Geschichte nicht bloß als Wandel, sondern als eine Art von Entwicklung zu denken. Aber lässt sich die Geschichte des Briefes und der Briefkultur tatsächlich als ein Prozess beschreiben, der mehr ist als eine Folge von einzelnen Phasen, die auch ganz anders hätte verlaufen können? Lässt sich, abstrakter formuliert, sagen, dass hier eine Struktur eine ,irreversible Steigerung“ erfährt - sei es, dass die Anzahl ihrer Elemente zunimmt, sei es, dass sich diese differenzieren, sei es, dass sie eine stärkere Ordnung gewinnen. ${ }^{62}$ Lässt sich im historischen Wandel etwas erkennen, das sich soziologisch als evolutionärer Prozess beschreiben lässt - mit Luhmann zu sprechen: als zunehmende Komplexität, zunehmende funktionale Spezifikation, zunehmende Normalisierung von Unwahrscheinlichkeit? ${ }^{63}$

Manches spricht dafür. Der Strukturwandel, den die Briefkultur im Kontext der literalen und literarischen Kultur des achtzehnten Jahrhunderts erfährt, trägt tatsächlich Züge einer solchen Entwicklung - zunächst deshalb, weil das Schreiben von Briefen hier in sozialer, zeitlicher und funktionaler Hinsicht entgrenzt wird. Immer mehr Leute schreiben immer häufiger aus immer mehr Anlässen, und dieser Prozess erzeugt eine Dynamik, in der sich die Bindung der Kommunikationsform Brief an verbindliche Gattungsnormen auflöst und der Brief als ein Medium mit eigenen, medienspezifischen Artikulationsstandards entdeckt wird. An die Stelle verbindlicher Musterbriefe tritt die Vielfalt individueller Stimmen und die als kulturbildend verstandene Idee, ihnen beim Schreiben von Briefen Raum zu ge-

59 Vgl. Wiedemann 2008.

60 Koselleck et al. 1975, 595.

61 Koselleck et al. 1975, 594-595.

62 Vgl. Bischof 2008, 159-161; zum Folgenden vgl. Vellusig 2011, 154-159.

63 Vgl. Luhmann 1985, 15-16. 
ben. Die funktionale Ausdifferenzierung einzelner, durch spezifische Anlässe und Kommunikationszusammenhänge geprägter Briefgenres wie dem Missionarsbrief oder dem Patientenbrief steht dem nicht entgegen - sie ist ein komplementärer Teil dieses Prozesses. ${ }^{64}$

Vielleicht war es immer schon ein Missverständnis, von der „Briefkultur der Empfindsamkeit“" zu sprechen und so die Kulturrevolution des achtzehnten Jahrhunderts zu einer Phase der Kulturgeschichte kleinzureden. Diese Kulturrevolution war die Folge einer strukturellen Verschriftlichung der gesellschaftlichen Wissensund Kommunikationsformen, die durch die Allianz von Presse und Post in Gang gesetzt wurde - „Aufklärung“ und „Empfindsamkeit“ sind dessen notdürftige Etiketten. Das achtzehnte Jahrhundert ist nicht deshalb das „klassische Jahrhundert des Briefes", ${ }^{65}$ weil in ihm mehr Briefe geschrieben worden wären als in den Jahrhunderten davor und danach, sondern weil das Schreiben von Briefen hier als eine Form des „geselligen Betragens“ (Schleiermacher) entdeckt und als ein way of life und eine Form der Lebenskunst betrieben wurde. Daher rührt denn auch die für die Zeitgenossen so naheliegende Vergegenwärtigung intimer Schreib- und Leseräume, die Jana Kittelmann in ihrem Beitrag nachzeichnet, oder der Vergleich des Briefs mit einem entspannten Plaudern und Schwatzen, auf den Ulrich Breuer und Anke Lindemann aufmerksam machen.

Es ist deshalb kein Zufall, dass die „Empfindsamkeit“ im Brief des neunzehnten Jahrhunderts kein Ende nehmen will: „Mit dem Ideal der Unmittelbarkeit entsteht ein Wunschbild, dessen Spuren im ganzen 19. Jahrhundert und im Grunde bis in die Gegenwart hinein nachzuweisen sind“, ${ }^{66}$ so der Befund von Renate Stauf, Annette Simonis und Jörg Paulus. Und: „Manche der im Band untersuchten Liebesbriefe des 19. und des 20. Jahrhunderts befinden sich liebesbrieftheoretisch betrachtet sehr viel näher an der Liebessprache und an subjektiven Selbstbildern des 18. Jahrhunderts als an jenen des Realismus oder der Moderne; für andere gilt das Umgekehrte." ${ }^{67}$ Empfindsamkeit, Romantik, Realismus und Moderne sind in briefgeschichtlicher Hinsicht durchaus fragwürdige, - wie der Beitrag von Wolfgang Bunzel zeigt - jedenfalls befragenswerte Kategorien.

Mit diesen skizzenhaften Hinweisen ist freilich noch keine Geschichte des Briefes geschrieben, aber doch eine Schwelle benannt, an die auch die Beiträge dieses Bandes immer wieder erinnern. Sie alle versuchen den einen oder anderen der hier angesprochenen Aspekte systematisch weiterzuführen oder nehmen sich

64 Reiches Anschauungsmaterial bieten die einschlägigen Beiträge des Handbuchs Brief. Vgl. statt anderer Delgado 2020; Schiegg 2020.

65 Steinhausen 1891, 302.

66 Vgl. Stauf et al. 2008, 7.

67 Stauf et al. 2008, 8. 
eine exemplarische Fallgeschichte vor - ob aus literaturwissenschaftlicher, geschichtswissenschaftlicher, sprachwissenschaftlicher oder theologischer Sicht. ${ }^{68}$ Auch wenn sich die einzelnen Beiträge nicht als Bausteine zu einer Geschichte des Briefs lesen lassen, verfolgen sie doch die Geschichtlichkeit ihres Gegenstandes, d. h. die historische Spezifik der jeweiligen Briefe und Korrespondenzen. Der Band steht der interdisziplinären Briefforschung und der interessierten Öffentlichkeit als Open Access Publikation zur Verfügung. Er lädt dazu ein, die mannigfaltigen Bezüge, die in dieser Einleitung allenfalls angedeutet wurden, in konstellierenden Lektüren selbst zu erkunden.

\section{Literatur}

Anderegg, Johannes (2001). Schreibe mir oft! Zum Medium Brief zwischen 1750 und 1830. Mit einem Beitrag v. Edith Anna Kunz. Göttingen.

Baasner, Rainer (1999). „Briefkultur im 19. Jahrhundert. Kommunikation, Konvention, Postpraxis“, in: Briefkultur im 19. Jahrhundert. Hg. v. Rainer Baasner. Tübingen: 1-36.

Bauer, Ingrid u. Christa Hämmerle (2017). „Liebe und Paarbeziehungen im ,Zeitalter der Briefe“ - ein Forschungsprojekt im Kontext“, in: Liebe schreiben. Paarkorrespondenzen im Kontext des 19. und 20. Jahrhunderts. Hg. v. Ingrid Bauer u. Christa Hämmerle. Göttingen: 9-47.

Bischof, Norbert (1993). „Untersuchungen zur Systemanalyse der sozialen Motivation I: Die Regulation der sozialen Distanz: Von der Feldtheorie zur Systemtheorie“, in: Zeitschrift für Psychologie 201: 5-43.

Bischof, Norbert (2008). Psychologie. Ein Grundkurs für Anspruchsvolle. Stuttgart.

Bohn, Cornelia (1997). „Ins Feuer damit: Soziologie des Briefgeheimnisses“, in: Schleier und Schwelle. Geheimnis und Öffentlichkeit. Hg. v. Aleida u. Jan Assmann. München: 41-51.

Bohnenkamp, Anne u. Waltraud Wiethölter (2008). „Zur Einführung“, in: Der Brief-Ereignis \& Objekt. Katalog der Ausstellung im Freien Deutschen Hochstift - Frankfurter Goethe Museum. Hg. v. Anne Bohnenkamp u. Waltraud Wiethölter. Frankfurt a. M.: IX-XI.

Bohrer, Karl Heinz (1987). Der romantische Brief. Die Entstehung ästhetischer Subjektivität. München u. Wien.

Bürgel, Peter (1976). „Der Privatbrief. Entwurf eines heuristischen Modells“, in: Deutsche Vierteljahrsschrift für Literaturwissenschaft und Geistesgeschichte 50: 281-297.

Buschmeier, Matthias (2014). „Pragmatische Literaturgeschichte. Ein Plädoyer“, in: Literaturgeschichte. Theorien - Modelle - Praktiken. Hg. v. Matthias Buschmeier, Walter Erhart u. Kai Kauffmann. Berlin u. Boston: 11-29.

68 Fast alle Beiträge des Bandes gehen auf Vorträge zurück, die auf zwei einander ergänzenden Tagungen gehalten wurden: Die erste (Nähe und Distanz. Elemente einer Anthropologie des Briefs, Karl-Franzens-Universität Graz, 3.-5. Oktober 2018) war den Kontinuitäten gewidmet, die in der Geschichte des Briefs sichtbar werden; die zweite (Die Geschichtlichkeit des Briefs. Kontinuität und Wandel einer Kommunikationsform, Philipps-Universität Marburg, 27. -28. Februar 2020) stand im Zeichen der Geschichtlichkeit des Briefs und seiner Formate. 
Chartier, Roger (1992). „Figures de l'auteur“, in: Ders.: L'ordre des livres. Lecteurs, auteurs, bibliothèques en Europe entre XIV et XVIII' siècle. Aix-en-Provence: 35-67.

Delgado, Mariano (2020). „Der Missionarsbrief“, in: Handbuch Brief. Von der Frühen Neuzeit bis zur Gegenwart. Hg. v. Marie Isabel Matthews-Schlinzig, Jörg Schuster, Gesa Steinbrink u. Jochen Strobel. Bd. 1: Interdisziplinarität - Systematische Perspektiven - Briefgenres. Berlin u. Boston: 484-490.

Dücker, Burckhard (1992). „Brief“, in: Literaturlexikon. Autoren und Werke deutscher Sprache. Hg. v. Walther Killy. Bd. 13: Begriffe, Realien, Methoden. Hg. v. Volker Meid. Gütersloh u. München: 124-129.

Dürscheid, Christa (2003). „Medienkommunikation im Kontinuum von Mündlichkeit und Schriftlichkeit. Theoretische und empirische Probleme“, in: Zeitschrift für Angewandte Linguistik 38: 37-56.

Ehlich, Konrad (1993). „Text und sprachliches Handeln. Die Entstehung von Texten aus dem Bedürfnis nach Überlieferung“, in: Schrift und Gedächtnis. Beiträge zur Archäologie der literarischen Kommunikation. Hg. v. Aleida u. Jan Assmann u. Christof Hardmeier. München: 24-43.

Foucault, Michel (1974). „Was ist ein Autor?“, in: Ders.: Schriften zur Literatur. Aus dem Französischen übersetzt v. Karin von Hofer. Frankfurt a. M.: 7-31.

Gabler, Thorsten (2020). „Theodor Fontanes Briefe“, in: Handbuch Brief. Von der Frühen Neuzeit bis zur Gegenwart. Hg. v. Marie Isabel Matthews-Schlinzig, Jörg Schuster, Gesa Steinbrink u. Jochen Strobel. Bd. 2: Historische Perspektiven - Netzwerke - Zeitgenossenschaften. Berlin u. Boston: 1231-1244.

Görner, Rüdiger (2008). „Brief über den Brief: Ein einführender Versuch“, in: Demnächst mehr Das Buch der Briefe. Deutschsprachige Briefe aus vier Jahrhunderten. Hg. v. Rüdiger Görner. Berlin: 15-41.

Gundelfinger, Friedrich [d. i. Friedrich Gundolf] (Hg.) (1907). Romantiker-Briefe. Jena.

Guntermann, Georg (1997). „Brief“, in: Literaturwissenschaftliches Lexikon. Grundbegriffe der Germanistik. Hg. v. Horst Brunner u. Rainer Moritz. Berlin: 54-56.

Hanneder, Jürgen (2016). „Der erste Indologe“. In: August Wilhelm Schlegel im Dialog. Epistolarität und Interkulturalität. Hg. v. Jochen Strobel. Paderborn: 67-80.

Henzel, Katrin (2020). „Materialität des Briefs“, in: Handbuch Brief. Von der Frühen Neuzeit bis zur Gegenwart. Hg. v. Marie Isabel Matthews-Schlinzig, Jörg Schuster, Gesa Steinbrink u. Jochen Strobel. Bd. 1: Interdisziplinarität - Systematische Perspektiven - Briefgenres. Berlin u. Boston: 222-231.

Heumann, Konrad (2020). „Der Brief als Sammlungsobjekt“, in: Handbuch Brief. Von der Frühen Neuzeit bis zur Gegenwart. Hg. v. Marie Isabel Matthews-Schlinzig, Jörg Schuster, Gesa Steinbrink u. Jochen Strobel. Bd. 1: Interdisziplinarität-Systematische Perspektiven - Briefgenres. Berlin u. Boston: 232-253.

Holly, Werner (1997). „Zur Rolle von Sprache in Medien. Semiotische und kommunikationsstrukturelle Grundlagen“, in: Muttersprache 1: 64-75.

Honold, Alexander u. Irmgard Wirtz (Hg.) (2019). Rilkes Korrespondenzen. Göttingen.

Kilian, Jörg (2005). Historische Dialogforschung. Eine Einführung. Tübingen.

Koschorke, Albrecht (1999). Körperströme und Schriftverkehr. Mediologie des 18. Jahrhunderts. München.

Košenina, Alexander (2002). „,Der wahre Brief ist seiner Natur nach poetisch‘. Vom Briefschreiber zum Autor - am Beispiel Hofmannsthals“, in: Autorschaft: Positionen und Revisionen. DFGSymposion 2001. Hg. v. Heinrich Detering. Stuttgart: 241-257. 
Koselleck, Reinhart, Christian Meier, Odilo Engels u. Horst Günther (1975). „Geschichte, Historie“. In: Geschichtliche Grundbegriffe. Historisches Lexikon zur politisch-sozialen Sprache in Deutschland. Hg. v. Otto Brunner, Werner Conze u. Reinhart Koselleck. Bd. 2. Stuttgart: 593-717.

Luckmann, Thomas (1988). „Kommunikative Gattungen im kommunikativen ,Haushalt‘ einer Gesellschaft“, in: Der Ursprung von Literatur. Medien, Rollen, Kommunikationssituationen zwischen 1450 und 1650. Hg. v. Gisela Smolka-Koerdt, Peter M. Spangenberg u. Dagmar Tillmann-Bartylla. München: 279-288.

Luhmann, Niklas (1985). „Das Problem der Epochenbildung und die Evolutionstheorie“, in: Epochenschwellen und Epochenstrukturen im Diskurs der Literatur- und Sprachhistorie. $\mathrm{Hg}$. v. Hans-Ulrich Gumbrecht u. Ursula Link-Heer. Frankfurt a. M.: 11-33.

Moran, William L. (Hg.) (1992). The Amarna Letters. Baltimore u. London.

Müller, Lothar (2012). Weiße Magie. Die Epoche des Papiers. München.

Müller, Wolfgang G. (1994). „Brief“, in: Historisches Wörterbuch der Rhetorik. Bd. 2. Hg. v. Gert Ueding. Tübingen: 60-76.

Müller, Wolfgang G. (2009). „Brief“, in: Handbuch der literarischen Gattungen. Hg. v. Dieter Lamping. Stuttgart: 75-83.

Neumann, Hans (2006). „Sumerische und akkadische Briefe des 3. Jt. v. Chr.“, in: Texte aus der Umwelt des Alten Testaments. Neue Folge. Hg. v. Bernd Janowski u. Gernot Wilhelm. Bd. 3: Briefe. Gütersloh: 1-20.

Reinard, Patrick (2016). Kommunikation und Ökonomie. Untersuchungen zu den privaten Papyrusbriefen aus dem kaiserzeitlichen Ägypten. 2 Bde. Rahden.

Sallaberger, Walther (1999). „Wenn Du mein Bruder bist, ... “. Interaktion und Textgestaltung in altbabylonischen Alltagsbriefen. Groningen.

Schiegg, Markus (2020). „Patientenbrief“, in: Handbuch Brief. Von der Frühen Neuzeit bis zur Gegenwart. Hg. v. Marie Isabel Matthews-Schlinzig, Jörg Schuster, Gesa Steinbrink u. Jochen Strobel. Bd. 1: Interdisziplinarität - Systematische Perspektiven - Briefgenres. Berlin u. Boston: 570-581.

Schmidt, Peter L. u. Hans Neumann (1997). „Brief“, in: Der Neue Pauly. Enzyklopädie der Antike. Hg. v. Hubert Cancik u. Helmuth Schneider. Bd. 2. Stuttgart u. Weimar: 771-775.

Schniedewind, William M. (Hg.) (2015). The El-Amarna Correspondence. A New Edition of the Cuneiform Letters from the Site of El-Amarna based on Collations of all Extant Tablets. Collated, Transcribed and Translated by Anson F. Rainey. 2 vols. Leiden u. Boston.

Schöne, Albrecht (1967). „Über Goethes Brief an Behrisch vom 10. November 1767“, in: Festschrift für Richard Alewyn. Hg. v. Herbert Singer u. Benno von Wiese. Köln u. Graz: 193-229.

Schöne, Albrecht (2015). Der Briefschreiber Goethe. München.

Schuster, Jörg u. Jochen Strobel (2013). „Briefe und Interpretationen. Über Möglichkeiten zu einer Geschichte der Briefkultur und über die Möglichkeit kulturhistorischer Skizzen mittels Brieflektüren“, in: Briefkultur. Texte und Interpretationen - von Martin Luther bis Thomas Bernhard. Hg. v. Jörg Schuster u. Jochen Strobel. Berlin u. Boston: XI-XXIV.

Schuster, Jörg (2014). „Kunstleben“. Zur Kulturpoetik des Briefs um 1900 - Korrespondenzen Hugo von Hofmannsthals und Rainer Maria Rilkes. Paderborn.

Siegert, Bernhard (1993). Relais. Geschicke der Literatur als Epoche der Post. 1751-1913. Berlin. Stauf, Renate, Annette Simonis u. Jörg Paulus (2008). „Liebesbriefkultur als Phänomen“, in: Der Liebesbrief. Schriftkultur und Medienwechsel vom 18. Jahrhundert bis zur Gegenwart. Hg. v. Renate Stauf, Annette Simonis u. Jörg Paulus. Berlin u. New York: 1-19. 
Steinhausen, Georg (1889/1891). Geschichte des deutschen Briefes. Zur Kulturgeschichte des deutschen Volkes. 2 Teile. Berlin.

Stiening, Gideon (2001): „Body-lotion. Körpergeschichte und Literaturwissenschaft“, in: Scientia Poetica 5: 183-215.

Strobel, Jochen (2018). „,Nach den ,Krisenjahren‘. August Wilhelm Schlegel in der europäischen Gelehrtenrepublik“, in: Zeitschrift für deutsche Philologie, 137, Sonderheft: August Wilhelm Schlegel und die Philologie. Hg. v. Matthias Buschmeier u. Kai Kauffmann. Berlin: 153-164.

Ter-Nedden, Gisbert (1988). „Das Ende der Rhetorik und der Aufstieg der Publizistik. Ein Beitrag zur Mediengeschichte der Aufklärung“, in: Soziale Welt, Sonderband 6: Kultur und Alltag. Hg. v. Hans-Georg Soeffner. Göttingen: 171-190.

Tschiggerl, Martin, Thomas Walach u. Stefan Zahlmann (2019). Geschichtstheorie. Wiesbaden. Ulbricht, Otto (2009). „Die Liebe des Ehrenfriedt Andreß Kien, 1716-1717“, in: Ders.: Mikrogeschichte. Menschen und Konflikte in der Frühen Neuzeit. Frankfurt a. M. u. New York: 206-255.

Vellusig, Robert (1991). „Mimesis von Mündlichkeit. Zum Stilwandel des Briefes im Zeitalter der technischen Reproduzierbarkeit der Schrift“, in: Medien und Maschinen. Literatur im technischen Zeitalter. Hg. v. Theo Elm u. Hans H. Hiebel. Freiburg i. Br.: 70-92.

Vellusig, Robert (2011). „Aufklärung und Briefkultur. Wie das Herz sprechen lernt, wenn es zu schreiben beginnt“, in: Das achtzehnte Jahrhundert 35,2: 154-171.

Vellusig, Robert (2018). „Die Poesie des Briefes. Eine literaturanthropologische Skizze“, in: Was ist ein Brief? Aufsätze zu epistolarer Theorie und Kultur / What is a letter? Essays on epistolary theory and culture. Hg. v. Marie Isabel Matthews-Schlinzig u. Caroline Socha. Würzburg: 57-75.

Vellusig, Robert (2020). „Literarische Anthropologie und Brief“, in: Handbuch Brief. Von der Frühen Neuzeit bis zur Gegenwart. Hg. v. Marie Isabel Matthews-Schlinzig, Jörg Schuster, Gesa Steinbrink u. Jochen Strobel. Bd. 1: Interdisziplinarität - Systematische Perspektiven Briefgenres. Berlin u. Boston: 254-268.

Vellusig, Robert (2021). „Imagination und Inszenierung. Symbolische Distanzregulation in der Briefkultur des 18. Jahrhunderts“, in: Briefe und Tagebücher zwischen Text und Quelle. Geschichts- und Literaturwissenschaft im Gespräch II. Hg. v. Volker Depkat u. Wolfram Pyta. Berlin: 145-182.

Wiedemann, Conrad (2008). „Die Liebesbriefe Friedrich Wilhelms II. von Preußen an Wilhelmine Enke“, in: Der Liebesbrief. Schriftkultur und Medienwechsel vom 18. Jahrhundert bis zur Gegenwart. Hg. v. Renate Stauf, Annette Simonis u. Jörg Paulus. Berlin u. New York: 61-80. 